\title{
Infinitely thin disks exhibit a first order nematic-columnar phase transition
}

\author{
Martin A. Bates and Daan Frenkel \\ FOM Institute for Atomic and Molecular Physics, Kruislaan 407, 1098 SJ Amsterdam, The Netherlands
}

(Received 14 November 1997)

\begin{abstract}
We have investigated the phase behavior of a system of hard cylinders in the limit $L / D \rightarrow 0$. Simulations in this limit are possible because the model can be mapped onto a system of particles with finite volume but variable shape. Our simulations indicate that infinitely thin disks exhibit a columnar phase, and that the nematic-columnar transition is first order. Combining this result with previous simulations of cut spheres, this transition appears to be first order for all aspect ratios $0<L / D \leqslant 0.1$. [S1063-651X(98)07904-5]
\end{abstract}

PACS number(s): 61.30.Cz, 64.70.Md

The majority of experimental, theoretical, and simulation investigations of liquid-crystal systems concentrate on elongated or rod-shaped molecules. However, disk-shaped molecules have also been shown to exhibit a variety of liquidcrystal phases [1]. Indeed, the formation of liquid crystals from disk-shaped molecules was anticipated in the 1920s [2], but the first example was not found until over 50 years later [3]. A number of models have been used to study the phase behavior of disk-shaped molecules at the microscopic level, in particular, infinitely thin platelets [4], oblate ellipsoids [5], and cut spheres [6]. Simulations of these hard-body models have shown that anisotropic excluded volume interactions alone can be responsible for the formation of aligned mesophases. However, platelets and ellipsoids exhibit only a nematic phase. The lack of a columnar phase in a system of ellipsoids has been accounted for by considering an affine transformation of the coordinates along the director, which maps a perfectly aligned nematic phase into a hard sphere fluid. This clearly excludes the possibility of observing liquid-crystal phases with partial translational order in such systems. Whereas the nematic phase can occur when the number density times the excluded volume is of order 1 , the columnar phase is only expected once the volume fraction of the disklike particles becomes appreciable. Indeed, in the early simulations of infinitely thin platelets [4], a nematic phase was observed, but a columnar phase was not. Veerman and Frenkel [6] showed that cut spheres exhibit a wider range of phase behavior, depending on the aspect ratio of the disk; they studied the cases $L / D=0.1,0.2$, and 0.3 , where $L$ is the thickness of the disk and $D$ is the diameter. The isotropic phase of the $L / D=0.3$ system was found to undergo a transition directly to a columnar phase. A cubatic phase, in which there is cubic orientational order but no translational order, was observed for $L / D=0.2$. Finally, a nematic phase was found between the columnar and isotropic phases for particles with aspect ratio $L / D=0.1$. For this model, the coexistence densities were determined to be $\rho_{N}^{*}=0.497$ and $\rho_{C}^{*}=0.546$, where $\rho^{*}$ is the density relative to the close packed density; this large density change indicates that the transition is quite strongly first order. The phase behavior of hard spherocylinders, the rod-shaped analog of this model, has now been extensively studied as a function of aspect ratio $[7,8]$ and, in particular, recent simulations suggest that the nematic-smectic transition in the limit $L / D \rightarrow \infty$ is first order [9]. In contrast, very little is known about the limiting behavior of the cut sphere model. We note that in the limit $D / L<<1$, there is no difference between a cut sphere and a cylinder of the same aspect ratio.

In this Brief Report, we examine the phase behavior of cylinders in the limit $L / D \rightarrow 0$. In an earlier study of infinitely thin disks [4], a columnar phase was not found because they were carried out at zero volume fraction. To extend the simulations to higher volume fractions, we employ a scaling argument similar to that introduced by Bolhuis and Frenkel [8] in their investigation of long rods in the limit $L / D \rightarrow \infty$. Our system is stretched by a factor $1 / L$ along the director, which increases the vanishingly small volume of a disk to a finite value. This permits us to consider the behavior of cylinders in the limit $L / D \rightarrow 0$ but $\rho L D^{2}$ finite. We note that, as for systems composed of rod-shaped particles, this scaling does not affect the reduced density $\rho^{*}\left(\equiv \rho / \rho_{\mathrm{CP}}\right)$, where $\rho_{\mathrm{CP}}$ is the close packed density, since $\rho_{\mathrm{CP}}$ scales in exactly the same manner as the number density $\rho$ and, while the reduced pressure $P^{*}\left(\equiv P / k_{B} T\right)$ is scaled by a factor $1 / L$, the ratio $P / \rho$ remains unchanged. However, the shape of the particles (see Fig. 1) is affected by the scaling.

We have determined the equation of state for infinitely thin disks using constant-pressure Monte Carlo simulations with a system of 720 scaled cylinders. The system was studied in two sequences; expansion of the columnar phase and compression of the nematic phase. The expansion series of

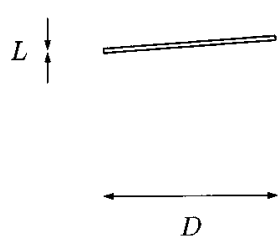

(a)

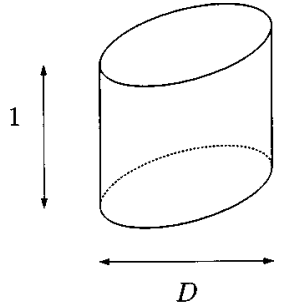

(b)
FIG. 1. Scaling of cut spheres or cylinders in the limit $L / D \rightarrow 0$. (a) A cylinder with finite $D$ but $L \rightarrow 0$; the tilt angle scales as $L / D$ [8]. (b) The disk scaled by a factor $1 / L$ along the $z$ axis. The scaled particle is a shifted cylinder with its axis aligned along $z$ and its faces tilted. 


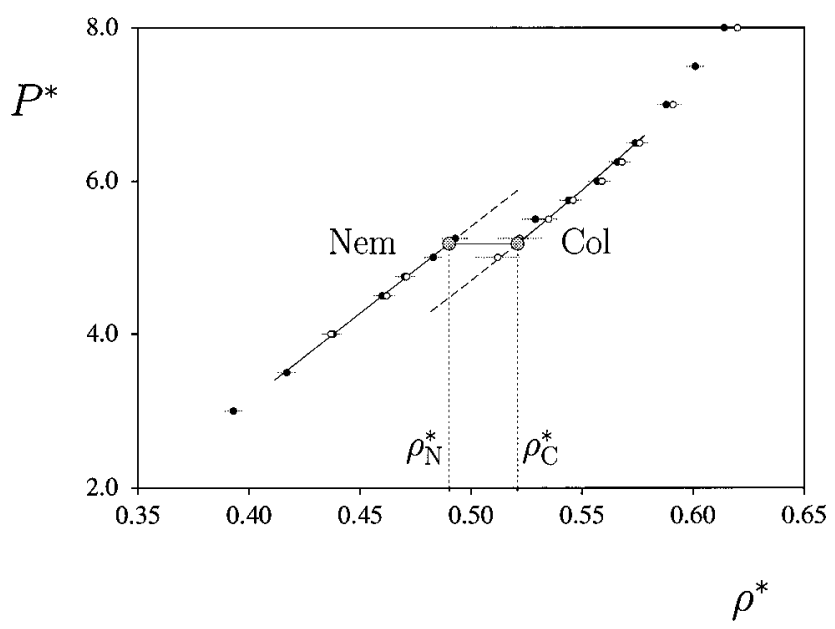

FIG. 2. Equation of state of the cylinder system in the limit $L / D \rightarrow 0$. The compression and expansion branches are plotted with filled and open circles, respectively. The large circles indicate the coexistence densities, as determined by the thermodynamic integration. The solid line indicates the equation of state in the vicinity of the transition. All quantities are plotted in reduced units: $\rho^{*} \equiv \rho / \rho_{\mathrm{CP}}$ and $P^{*} \equiv P / k_{B} T$ (see text).

simulations was started from a close packed $A A A$ stacked hexagonal lattice, which was equilibrated at a reduced pressure $P^{*}=15.0$. The system was then expanded by gradually reducing the pressure. A nematic configuration was prepared by expanding the $A A A$ stacked hexagonal lattice to a reduced density $\rho^{*}=0.30$. This system was then gradually compressed by increasing the pressure from $P^{*}=3.0$ in small steps. In each series of simulations, the initial configuration was perfectly aligned along the $z$ axis. A Monte Carlo move was performed by selecting a pair of random particles, $i$ and $j$, and giving each a random displacement; in addition, a random tilt was applied to particle $i$ and an equal but opposite tilt was applied to particle $j$. This ensures that the director orientation does not change, and, therefore, that our scaling argument remains valid.

The equation of state, shown in Fig. 2, clearly indicates a first order transition between the nematic and columnar phases. In order to locate the transition accurately, we have used a thermodynamic integration scheme to determine the free energy difference between the nematic and columnar phases. For this we require a reversible path between the two phases. To achieve this, we apply a perturbation

$$
H_{\lambda}=\lambda \sum_{i} \cos \left(\frac{2 \pi n_{x} x_{i}}{l_{x}}\right) \sin \left(\frac{\pi n_{y} y_{i}}{l_{y}}\right)
$$

to the system, where $l_{x}$ and $l_{y}$ are the $x$ and $y$ dimensions of the box, $x_{i}$ and $y_{i}$ are the $x$ and $y$ coordinates of particle $i$, and $n_{x}$ and $n_{y}$ are the number of columns in the $x$ and $y$ directions. By increasing the parameter $\lambda$, the nematic phase can undergo a continuous transition to the columnar phase at constant density. The difference in the Helmholtz free energy between the nematic phase at a density $\rho_{1}^{*}$ and the columnar phase at a density $\rho_{2}^{*}$ is given by

$$
\begin{aligned}
\frac{\Delta F}{N}= & \int_{0}^{\lambda_{\max }}\left\langle\cos \left(\frac{2 \pi n_{x} x_{i}}{l_{x}}\right) \sin \left(\frac{\pi n_{y} y_{i}}{l_{y}}\right)\right\rangle_{\rho_{2}^{*}} d \lambda \\
& -\int_{0}^{\lambda_{\max }}\left\langle\cos \left(\frac{2 \pi n_{x} x_{i}}{l_{x}}\right) \sin \left(\frac{\pi n_{y} y_{i}}{l_{y}}\right)\right)_{\rho_{1}^{*}} d \lambda \\
& -\int_{\rho_{1}^{*}}^{\rho_{2}^{*} P_{\lambda_{\max }}(\rho)} d \rho,
\end{aligned}
$$

where $\lambda_{\max }$ is chosen such that the first order transition between the phases is completely suppressed and the angular brackets $\langle\cdots\rangle$ indicate an average over molecules and configurations. Two series of constant-volume simulations were performed to evaluate numerically the first two integrals of Eq. (2) at densities $\rho_{1}^{*}=0.47$ and $\rho_{2}^{*}=0.55$, in which the value of $\lambda$ was gradually increased from 0 to $\lambda_{\max }=3.0$. A further series of constant-pressure simulations was used to determine the equation of state $P_{\lambda_{\max }}(\rho)$ for the system with the perturbation (1) applied with $\lambda$ set equal to $\lambda_{\max }$, covering the range of densities between $\rho_{1}^{*}$ and $\rho_{2}^{*}$; in turn, this was used to evaluate the final integral of Eq. (2). Our calculations gave the Helmholtz free-energy difference between the nematic and columnar phases at $\rho_{1}^{*}=0.47$ and $\rho_{2}^{*}=0.55$, respectively, to be $\Delta F / N=0.992(10)$. The pressure dependence of the free energy, relative to that of the nematic phase at $\rho_{1}^{*}=0.47$, was then determined for both the nematic and columnar phases by integration of the appropriate branch of the equation of state. At the point of coexistence, the Gibbs free energies of the two phases are equal; our calculations lead to values for the coexistence densities of $\rho_{N}^{*}=0.490(5)$ and $\rho_{C}^{*}=0.521(5)$.

We have used a scaling argument to show that the cut sphere model for disk-shaped liquid crystals exhibits a columnar phase in the limit $L / D \rightarrow 0$. This result contrasts that of a previous study of infinitely thin disks [4], where a columnar phase was not observed because of the vanishing excluded volume interaction as two particles become perfectly aligned. The coexistence densities determined for cylinders in the limit $L / D \rightarrow 0 \quad\left(\rho_{N}^{*}=0.490, \rho_{C}^{*}=0.521\right)$ are similar to those for a system of cut spheres of aspect ratio $L / D=0.1\left(\rho_{N}^{*}=0.497, \rho_{C}^{*}=0.546\right)[6]$, and, although the transition in our system appears to be slightly weaker, the transition is clearly first order. We conclude that the transition between the nematic and columnar phases of cut spheres almost certainly remains first order for all aspect ratios in the range $0<L / D \leqslant 0.1$.

We are grateful to Blandine Jérôme and Ronald Blaak for critical readings of the manuscript. The work of the FOM Institute is part of the research program of FOM (Stichting Fundamenteel Onnderzoek der Materie), and is supported by NWO (Nederlandse Organisatie voor Wetenschappelijk Onderzoek). M.A.B. acknowledges the financial support of the EU through the Marie Curie TMR Fellowship Programme (Contract No. ERB4001GT965174). 
[1] S. Chandrasekhar, Philos. Trans. R. Soc. London, Ser. A 309, 93 (1983).

[2] D. Vorländer, Z. Phys. Chem. (Munich) 105, 211 (1923).

[3] S. Chandrasekhar, B. K. Sadashiva, and K. A. Suresh, Pramana 9, 471 (1977).

[4] R. Eppenga and D. Frenkel, Mol. Phys. 52, 1303 (1984).

[5] D. Frenkel and B. M. Mulder, Mol. Phys. 55, 1171 (1985).
[6] J. A. C. Veerman and D. Frenkel, Phys. Rev. A 45, 5632 (1992).

[7] S. C. McGrother, D. C. Williamson, and G. Jackson, J. Chem. Phys. 104, 6755 (1996).

[8] P. Bolhuis and D. Frenkel, J. Chem. Phys. 106, 666 (1997).

[9] J. M. Polson and D. Frenkel, Phys. Rev. E 56, 6260 (1997). 